\title{
Intravenous lipid emulsion therapy for cardiac arrest and refractory ventricular tachycardia due to multiple herb intoxication
}

\author{
Sangsoo Han, Hoon Lim, Hyun Noh \\ Department of Emergency Medicine, Soonchunhyang University Bucheon Hospital, Soonchunhyang \\ University College of Medicine, Bucheon, Korea
}

Herbal products have been used for therapeutic purposes for a long time. However, many herbs can be toxic and even life-threatening. If refractory ventricular tachycardia (VT) is caused by herbal products and shows no response to conventional therapy, intravenous lipid emulsion (ILE) therapy can be considered. We report a case of herbal intoxication leading to refractory VT, which was successfully treated with ILE therapy. A 36-year-old woman with aplastic anemia presented with mental changes. She had taken an unknown herbal decoction three days before visiting the hospital. Soon after coming to the hospital, she went into cardiac arrest. Cardiopulmonary resuscitation was performed, and return of spontaneous circulation with VT was achieved. Synchronized cardioversion was then performed and amiodarone was administered. However, VT with pulse continued, so ILE therapy was attempted, which led to the resolution of VT.

Keywords Herb-drug interactions; Fat emulsions, intravenous; Tachycardia, ventricular; Heart arrest; Toxicity
elSSN: 2383-4625

Received: 4 October 2018

Revised: 24 October 2018

Accepted: 15 November 2018

Correspondence to: Hyun Noh Department of Emergency Medicine, Soonchunhyang University Bucheon Hospital, Soonchunhyang University College of Medicine, 170 Jomaru-ro, Wonmi-gu, Bucheon 14584, Korea E-mail: dingii@schmc.ac.kr ORCID

http://orcid.org/0000-0003-4078-333X

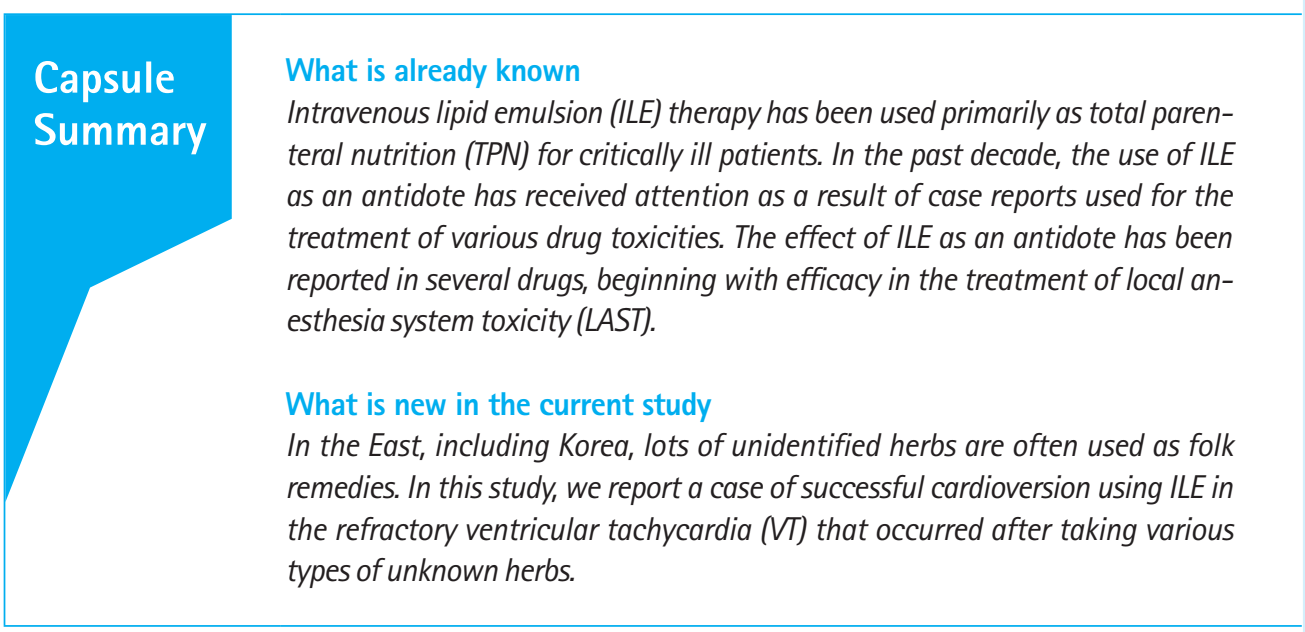

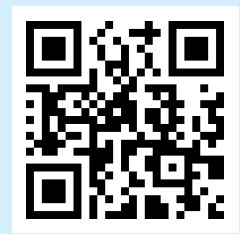

How to cite this article:

Han S, Lim H, Noh H. Intravenous lipid emulsion therapy for cardiac arrest and refractory ventricular tachycardia due to multiple herb intoxication. Clin Exp Emerg Med 2019;6(4):366-369.

This is an Open Access article distributed under the terms of the Creative Commons Attribution Non-Commercial License (http:// creativecommons.org/licenses/by-nc/4.0/). 


\section{INTRODUCTION}

Herbal products have been used for therapeutic purposes for a long time. ${ }^{1}$ In the Asian region, a variety of herbs, of which therapeutic or side effects are unclear, are used because of the influence of Oriental medicine. However, some of these herbs may be toxic or even fatal for various organs including the heart, lung, kidney, liver, brain, and others. ${ }^{2}$

Lipid emulsion has been developed for use as part of total parenteral nutrition in malnutrition patients and has recently been used effectively as an antidote to lipophilic drug intoxication. ${ }^{3}$ In particular, it is widely used for the treatment of toxicity by local anesthetics and can also be used for intractable cardiovascular collapse by non-local anesthetics with no specific antidote. ${ }^{4}$

We report a case of intravenous lipid emulsion (ILE) therapy used as a treatment in refractory ventricular tachycardia (VT) with cardiac collapse. We believe this case is the first report of ILE therapy in multiple unknown herbal poisoning.

\section{CASE REPORT}

This study was approved by the institutional review board of the Soonchunhyang University Bucheon Hospital (2018-08-011). Written informed consent was obtained from the patient for their anonymized information to be published in this article.

A 36-year-old female visited the outpatient clinic for a routine follow-up and suffered an episode of loss of consciousness as she was leaving the hospital. She had a history of bone marrow trans- plantation due to aplastic anemia 6 months ago and currently not taken any medication. She was then transferred to the emergency department, with a Glasgow Coma Scale score of 6 (eyeopening response 1 , best verbal response 1 , best motor response 4). Initial blood pressure was $110 / 80 \mathrm{mmHg}$ and pulse was 120 bpm. However, cardiac arrest occurred 5 minutes after arriving at the emergency department, and cardiopulmonary resuscitation (CPR) was performed immediately. The initial arrest rhythm was pulseless VT, so defibrillation with $200 \mathrm{~J}$ was performed. Spontaneous circulation returned after one cycle of CPR; however, a sustained return of spontaneous circulation (ROSC) was not achieved. The total CPR time was 12 minutes and three defibrillation attempts were performed. Three 1-mg epinephrine doses and 300 $\mathrm{mg}$ of amiodarone were administered. When ROSC was finally achieved, the electrocardiogram showed VT with pulse (Fig. 1). Her vital signs after ROSC were as follows: blood pressure was $60 / 40 \mathrm{mmHg}$, pulse was $200 \mathrm{bpm}$, body temperature was $36.4^{\circ} \mathrm{C}$, and $\mathrm{SpO}_{2}$ was $79 \%$. Synchronized cardioversion was performed at $100 \mathrm{~J}$ and $150 \mathrm{mg}$ of amiodarone were administered. VT with pulse developed again less than half an hour later after rhythm conversion, so we decided to apply extracorporeal membrane oxygenation (ECMO). ECMO was applied 40 minutes after arrival at the emergency department.

The patient's initial laboratory findings were as follows: white blood cell count $8.2 \times 10^{3} / \mu \mathrm{L}$, hemoglobin $12.1 \mathrm{~g} / \mathrm{dL}$, platelet count $149 \times 10^{3} / \mu \mathrm{L}$, blood urea nitrogen $6.1 \mathrm{mg} / \mathrm{dL}$, creatinine $1.0 \mathrm{mg} / \mathrm{dL}$, aspartate aminotransferase $129 \mathrm{IU} / \mathrm{L}$, alanine aminotransferase $81 \mathrm{IU} / \mathrm{L}$, creatinine-kinase $87 \mathrm{IU} / \mathrm{L}$, total bilirubin $0.77 \mathrm{mg} / \mathrm{dL}$, C-

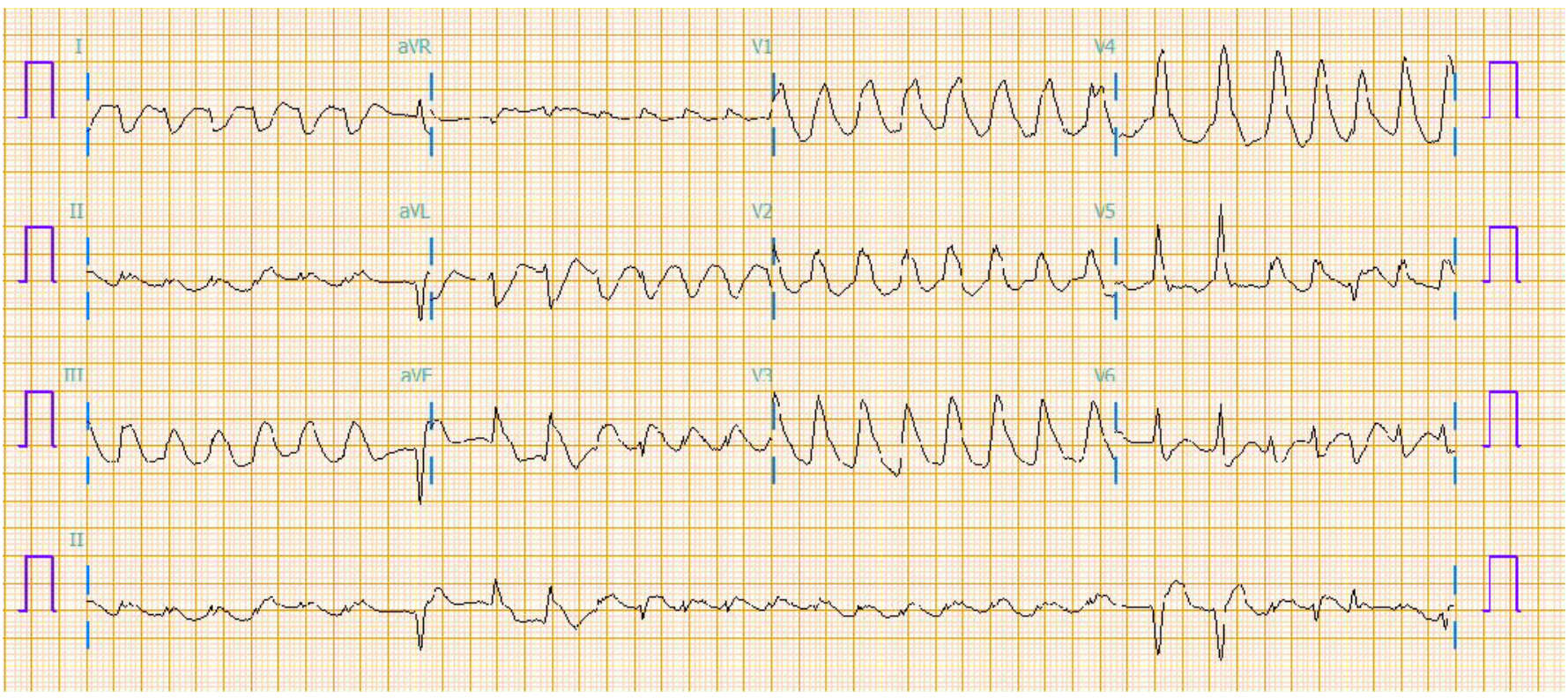

Fig. 1. Electrocardiogram rhythm after return of spontaneous circulation. 


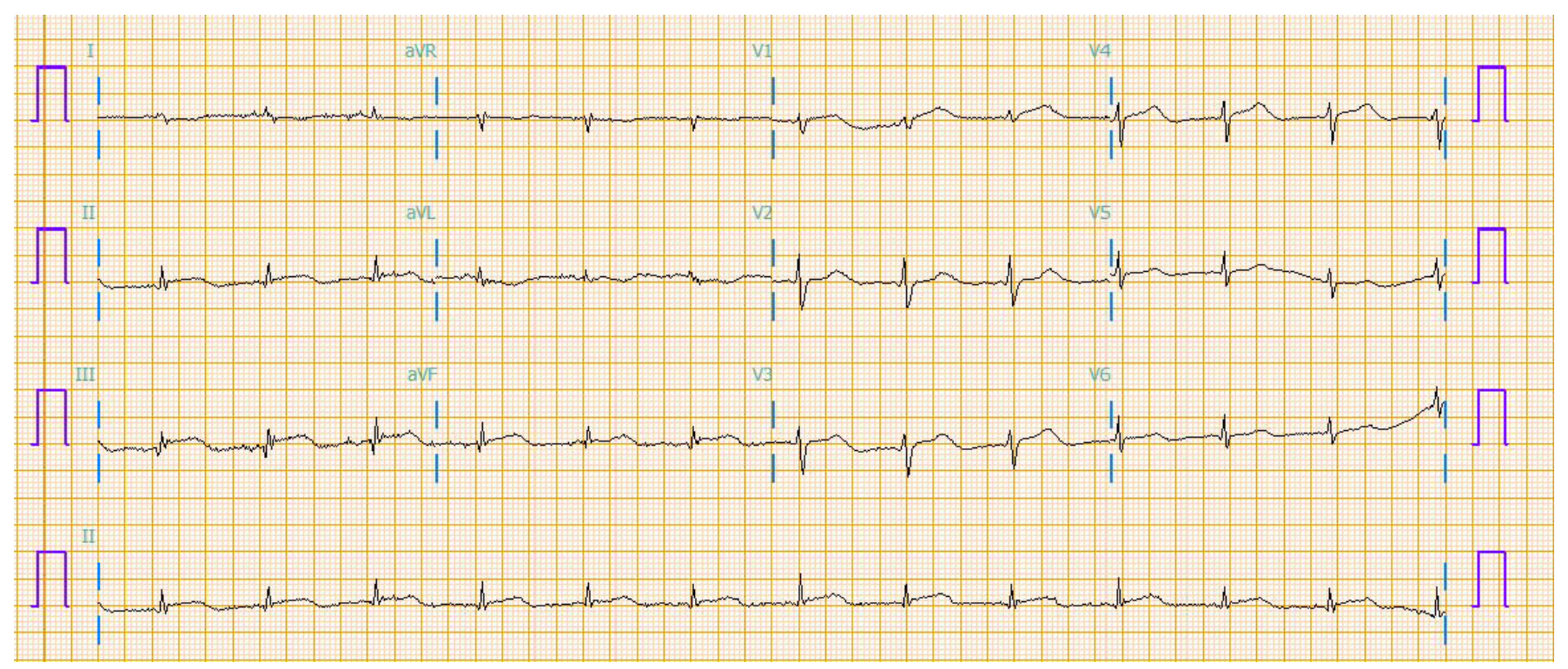

Fig. 2. Electrocardiogram rhythm after intravenous lipid emulsion therapy.

reactive protein $0.22 \mathrm{mg} / \mathrm{dL}$, sodium $145 \mathrm{mmol} / \mathrm{L}$, potassium 5.6 $\mathrm{mmol} / \mathrm{L}$, chlorine $101 \mathrm{mmol} / \mathrm{L}$, magnesium $2.8 \mathrm{mg} / \mathrm{dL}$, total calcium 7.9 mg/dL, CK-MB 72.2, myoglobin 7540, troponin I 10.81, T3 $0.84 \mathrm{ng} / \mathrm{mL}$, free T4 $1.36 \mathrm{ng} / \mathrm{dL}$, and thyroid-stimulating hormone $0.73 \mathrm{ulU} / \mathrm{mL}$.

A total of $20 \mathrm{~mL}$ of $10 \%$ calcium gluconate and $100 \mathrm{mEq}$ of bicarbonate were administered to correct hyperkalemia. Coronary angiography was done 1 hour and 40 minutes after the arrival at the emergency department, which showed normal coronary arteries. She was admitted to the intensive care unit after angiography, and VT with pulse continued for 3 hours at intervals of 30 minutes to 1 hour.

To find out the cause of refractory VT, further history taking was done with her father. According to him, he had heard from a neighbor that herbs were useful for treating aplastic anemia, so he bought multiple herbs for the patient from a market a few days before. The patient had been taking a herbal decoction for three days before visiting the hospital. However, he did not know the exact name of the herbs. We then considered ILE therapy as another treatment option. Thus, a 100-mL bolus of 20\% ILE (Smoflipid) was injected and sinus rhythm was achieved within 3 minutes of administration (Fig. 2). VT did not return; therefore, no additional ILE was administered. She was weaned off ECMO after three days and discharged with a Cerebral Performance Category score of 1 (full recovery) after 15 days without any complications.

\section{DISCUSSION}

In Korea, herbs and plants can be easily bought in traditional markets. However, herb markets are not strictly regulated and research on herbal safety is insufficient, with their exact effects remaining unknown. Herbs can cause side effects when misused and some, like morning glory, jimsonweed, or yohimbe can cause euphoric, stimulant, or hallucinogenic effects. ${ }^{5}$ From 1915 to 1990 , there were 2,788 cases of side effects associated with 460 different herbs reported in 408 Chinese medical journals. In addition, reports of herbal toxicity are increasing. ${ }^{6}$ Additionally, some herbs can be toxic to certain organs. Aconite, for example, can induce cardiac failure due to its cardiac toxicity. ${ }^{7}$ Because of herbal interactions, serious damage can be caused if multiple herbs are consumed at once without caution. ${ }^{5}$ We were unable to identify which herbs the patient ingested; however, Angelica gigas Nakai, Cnidium officinale Makino, Citrus unshiu Markovich, and Glycyrrhiza uralensis Fischer are thought to be useful for anemia in Korea, so it can be speculated that the patient could have ingested these herbs. Angelica gigas Nakai is known to reduce cardiac contraction, ${ }_{1}^{8}$ but there are no reports that it causes arrhythmia; the three other herbs also lack evidence of cardiac toxicity. Therefore, she might have taken additional herbs and one or more of them could have caused the refractory VT.

ILE has been widely used as an antidote to local anesthetics intoxication, and its effect on treatment for poisoning induced by antipsychotics, antidepressants, or calcium channel blockers has recently been found. ${ }^{9}$ The main mechanism of action of ILE is as a lipid sink, which means that a sufficient amount of lipids binds to toxins in tissues and separates them. In addition, ILE might directly increase intramyocyte calcium level and lead to a direct positive inotropic effect. ${ }^{10}$ It is known that ILE therapy is useful for lipophilic xenobiotic poisoning, and there have been reports 
about its effect on various drug intoxications over recent years. ${ }^{9}$ Also, some studies suggest that ILE may act as a useful antidote for cardiovascular collapse caused by lipophilic drug overdose., ${ }^{4,11}$ However, even with the increasing applications of ILE therapy, its precise indications are yet to be established. ${ }^{9}$ Most of the existing literature recommends ILE therapy in case of life-threatening cardiovascular toxicity that is nonresponsive to conventional therapy. ${ }^{9}{ }^{12}$ However, it is inadequate for first-line therapy since ILE may enhance absorption of toxins or inhibit the activity of other drugs like vasopressors..$^{13}$ In our case, synchronized cardioversion and administration of antiarrhythmics were performed first, but they were not effective, so ILE therapy was performed as adjunctive treatment.

VT can be caused by myocardial infarction; valvular heart disease; cardiomyopathy; electrolyte imbalance; and intoxication by drugs such as digoxin, procainamide, and quinidine. Herbal poisoning by aconite can also cause $\mathrm{VT} .{ }^{14}$ The aim of $\mathrm{VT}$ treatment is primarily to correct the underlying cause such as ischemia or electrolyte imbalance, and if VT persists, antiarrhythmics such as amiodarone can be used or ablation can be performed. In our case, VT persisted despite the correction of potassium levels and the use of amiodarone. Therefore, ILE therapy was performed on the assumption that cardiotoxicity could have been caused by lipophilic components in herbs that the patient consumed. UltimateIy, ILE therapy was effective and no more VT occurred.

The limitation of this case report is that it is not possible to know which herb caused refractory VT because the patient was taking multiple herbs at once. Therefore, it is difficult to determine which herb responded to ILE therapy. However, since the patient was not taking any medications before and VT suddenly happened after the ingestion of multiple herbs, it is very likely that herbs were the cause of VT. Also, there is a strong possibility that there are toxic lipophilic substances in herbs because ILE therapy was shown to be effective. ${ }^{15}$ ILE therapy should be considered as an adjunctive treatment if refractory $\mathrm{VT}$ in multiple unknown herbal intoxication is not treated successfully with standard therapy.

\section{CONFLICT OF INTEREST}

No potential conflict of interest relevant to this article was reported.

\section{REFERENCES}

1. Williamson EM. Drug interactions between herbal and prescription medicines. Drug Saf 2003;26:1075-92.

2. Tovar RT. Clinical approach to clinical herbal toxicity. Semin Diagn Pathol 2009;26:28-37.

3. Damitz $R$, Chauhan A. Parenteral emulsions and liposomes to treat drug overdose. Adv Drug Deliv Rev 2015;90:12-23.

4. Weinberg G. Lipid infusion resuscitation for local anesthetic toxicity: proof of clinical efficacy. Anesthesiology 2006;105:7-8.

5. Richardson WH 3rd, Slone CM, Michels JE. Herbal drugs of abuse: an emerging problem. Emerg Med Clin North Am 2007; 25:435-57.

6. Ko RJ. Causes, epidemiology, and clinical evaluation of suspected herbal poisoning. J Toxicol Clin Toxicol 1999;37:697708.

7. Fatovich DM. Aconite: a lethal Chinese herb. Ann Emerg Med 1992;21:309-11.

8. Oh SR, Ok S, Jung TS, et al. Protective effect of decursin and decursinol angelate-rich Angelica gigas Nakai extract on dextran sulfate sodium-induced murine ulcerative colitis. Asian Pac J Trop Med 2017;10:864-70.

9. Lam SH, Majlesi N, Vilke GM. Use of intravenous fat emulsion in the emergency department for the critically ill poisoned patient. J Emerg Med 2016;51:203-14.

10. Rothschild L, Bern S, Oswald S, Weinberg G. Intravenous lipid emulsion in clinical toxicology. Scand J Trauma Resusc Emerg Med 2010;18:51.

11. Rosenblatt MA, Abel M, Fischer GW, Itzkovich CJ, Eisenkraft JB. Successful use of a $20 \%$ lipid emulsion to resuscitate a patient after a presumed bupivacaine-related cardiac arrest. Anesthesiology 2006;105:217-8.

12. St-Onge $M$, Anseeuw $K$, Cantrell FL, et al. Experts consensus recommendations for the management of calcium channel blocker poisoning in adults. Crit Care Med 2017;45:e306-15.

13. Gosselin S, Hoegberg LC, Hoffman RS, et al. Evidence-based recommendations on the use of intravenous lipid emulsion therapy in poisoning. Clin Toxicol (Phila) 2016;54:899-923.

14. Lowe L, Matteucci MJ, Schneir AB. Herbal aconite tea and refractory ventricular tachycardia. N Engl J Med 2005;353:1532.

15. Tamir S, Eizenberg M, Somjen D, Izrael S, Vaya J. Estrogen-like activity of glabrene and other constituents isolated from licorice root. J Steroid Biochem Mol Biol 2001;78:291-8. 